\title{
Prosthetic rehabilitation of an edentulous cleft palate using a denture with a palatal obturator: a clinical report
} \author{
Alberto Albaladejo ${ }^{1}$, R. Del Castillo ${ }^{5}$, J. Maroto ${ }^{5}$. \\ ${ }^{1}$ Tenured lecturer. Department of Surgery. Faculty of Medicine. University of Salamanca. Spain. \\ ${ }^{2}$ Graduate in Dentistry. Postgraduate student. University of Salamanca. Spain. \\ ${ }^{3}$ Graduate in Dentistry. Postgraduate student. University of Granada. Spain. \\ ${ }^{4}$ Associate Professor. Department of Surgery. Faculty of Medicine. University of Salamanca. Spain \\ ${ }^{5}$ Tenured lecturer. Department of Stomatology. Faculty of Dentistry. University of Granada. Spain.
}

Javier Montero ${ }^{1}$, Carla Macedo ${ }^{2}$, Manuel Rodriguez ${ }^{3}$, Antonio López-Valverde ${ }^{4}$, Rafael Gómez de Diego ${ }^{4}$,

Correspondence:

Clínica Odontológica. Facultad de Medicina,

Departamento de Cirugía. C/Alfonso X el Sabio S/N.

C.P. 37007. Salamanca. Spain.

E-mail: javimont@usal.es

Received: 26/01/2011 Accepted: $27 / 03 / 2011$
Montero J, Macedo C, Rodriguez M, López-Valverde A, Gómez de Diego R, Albaladejo A, Del Castillo R, Maroto J. Prosthetic rehabilitation of an edentulous cleft palate using a denture with a palatal obturator: a clinical report. J Clin Exp Dent. 2011;3(Supp11):e365-8 http://www.medicinaoral.com/odo/volumenes/v3iSuppl1/jcedv3iSuppl1p365.pdf

Article Number: $50495 \quad$ http://www medicinaoral com/odo/indice.htm C Medicina Oral S. L. C.I.F. B 96689336 - eISSN: 1989-5488

eMail: jced@jced.es

\begin{abstract}
A cleft palate is a genetic disorder that occurs when an oro-nasal communication is present between the palate and the base of the nose. During pregnancy, the maxillary is not completely merged, and the defect is only seen at birth. Possible causes are hormonal imbalances, nutritional deficiencies, infections, radiation during pregnancy, alcohol or cigarette consumption, the ingestion of other teratogenic substances by the mother, and heredity. The resulting defect requires corrective surgery. In the case of a cleft palate, surgery is postponed until after the first year of life to avoid disturbing the normal development of speech and the risk of aspiration of food, which causes infections such as otitis and pneumonia. Ear infections can harm the development of speech in cases where surgery is not possible or the defect has reappeared. This article describes the prosthetic rehabilitation of a edentulous patient: a woman of 53 years old with a cleft palate who was treated surgically. In a clinical examination, a residual palatal defect was identified. The prosthetic rehabilitation involved the emplacement of a complete adapted prosthesis, using a palatal obturator, with a view to sealing the defect and allowing the patient to acquire better speech quality, and improve her nutrition and well-being.
\end{abstract}

Key words: complete denture, cleft palate, cleft lip, obturator. 


\section{Introduction}

Although cleft lips and palates are not regularly seen in general dental practice, their number is not negligible. These congenital anomalies are quite frequent although their prevalence among the general population depends on racial, ethnic and geographic factors, as well as on socio-economic status. It has been estimated to range from 1:500 to 1:2500 live births (1). Cleft lips occur in $20-30 \%$ of cases; a cleft lip and palate in $35-50 \%$, and cleft palate alone in $30-45 \%$ (2).

The difficulties of cleft palate patients involve physical activities such as eating, breathing and speaking, but their psychological well-being is also affected. Thus, it has been suggested that a prosthesis may improve both the physical and psychological performances of patients, as well as their quality of life (3).

The prosthetic rehabilitation of patients with a cleft lip or palate requires a multidisciplinary team of professionals so that long-term success in treatment can be achieved. Plastic surgeons, orthodontists, and prosthodontists are only part of the therapeutic team responsible for the medical care, which in many cases begins soon after birth and continues along the various stages of patients' lives until they reach maturity (4).

There are other complex cases of cleft palate involving function, aesthetics and phonetics that require a more invasive restorative intervention. However, an alternative conservative treatment can be sought in conventional prostheses for patients who choose not to undergo surgery (4). Complete dentures are especially indicated in patients with a tissue deficiency, several fistulae, soft palate dysfunctions, or uncoordinated nasopharyngeal sphincter action, which can lead to hypernasal speech (5). Prosthodontic care has a long and rich history in the care of patients with cleft lips and palates. With increased knowledge of craniofacial growth and development and improved surgical and orthodontic treatment, today's cleft palate/lip patients receive better care, and in less time (5).

This clinical report describes the rehabilitation of a cleft palate patient using a complete denture with a palatal obturator.

\section{Case Report}

A 53-year-old woman came to the Patient Admission Office at the School of Dentistry of the University of Granada seeking prosthetic treatment for her perforated maxilla. She was referred to the Prosthetic Master's Degree team of the same School for her rehabilitation needs to be assessed. She referred to several previous attempts to wear conventional dentures, which were not tolerated because of loss of retention. The patient showed marked bone atrophy (Fig. 1) and a total absence of teeth in her maxilla. Interventions to reduce her palatal cleft lip/palate were performed in 1982 and later,

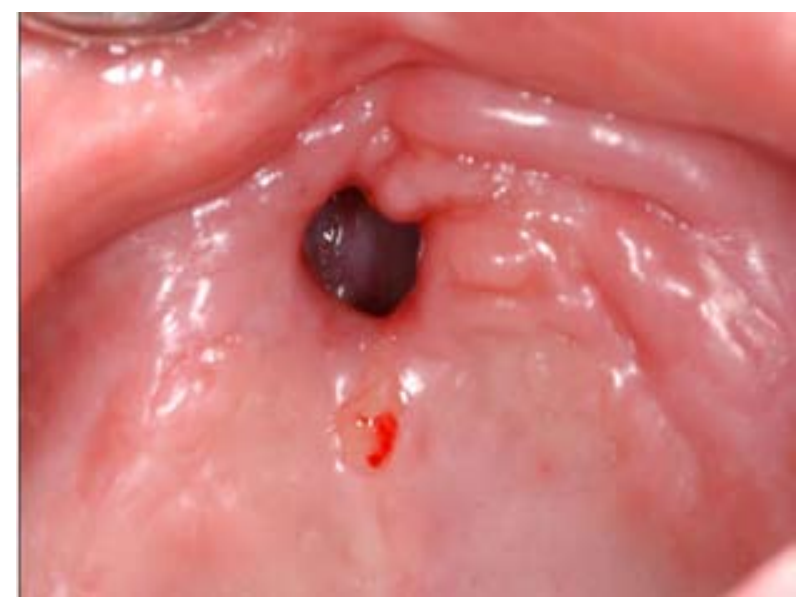

Fig. 1. View of the maxilla with a cleft palate.

in 1998, the patient underwent reconstructive surgery with rhinoseptoplasty with grafts of cartilage to improve her breathing. However, no intervention was directed towards increasing her oral function (mainly eating and speaking), and she was not able to wear a conventional denture because of the severe atrophy. Thus, prosthetic treatment was chosen, elaborating a removable acrylic prosthesis best suited for her, with a palatal obturator of soft resin to improve denture retention and stability. For economic reasons, the patient refused the placement of an implant-retained overdenture.

After preliminary impressions of the maxillary and mandibular complete arches had been made using irreversible hydrocolloid impression material (alginate), custom trays were made, adding a loop-shaped retainer made of $0.8-\mathrm{mm}$ steel to the maxillary tray to support the silicone and reproduce the palatal defect (Fig. 2).

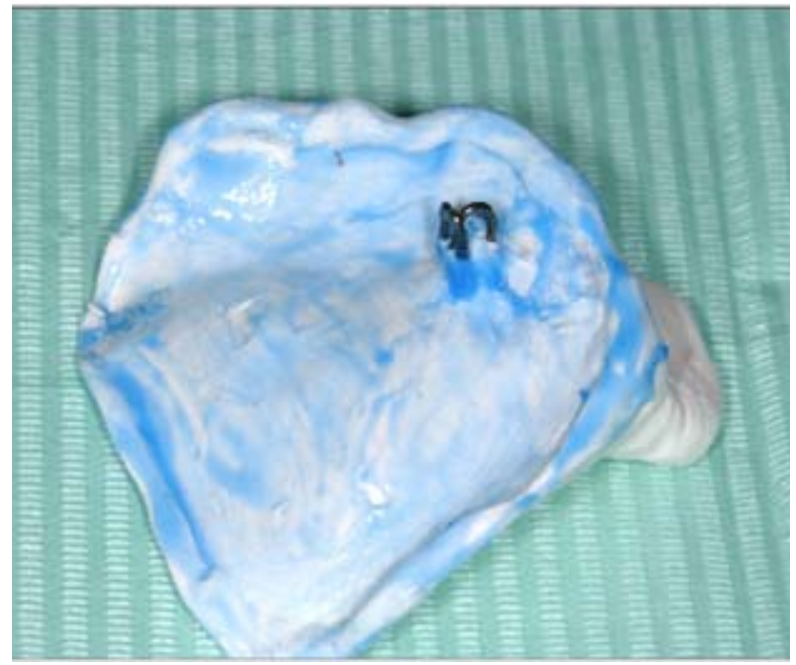

Fig. 2. Steel retainer in position.

A complete denture of acrylic was made using routine procedures, but incorporating a retainer (called an obturator) to support the closure of the palatovelar communication. It was initially made of a permanent soft e366 
silicone-based relining material (Ufi Gel SC,VOCO ${ }^{\mathrm{TM}}$, Germany) and was retained by the loop-shaped steel retainer, which entered the palatal hole and increased the retention of the denture.

After an adaptation period of one month, the silicone obturator was transformed into acrilic using a permanent hard relining material that set intraorally (Ufi

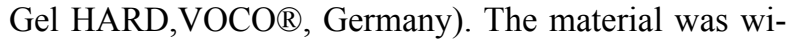
thdrawn from the mouth before it had fully set to cut off excess material and shape it into a retentive but not harmful design. To avoid dehiscence of the antrum, we checked that harmful pressure forces were properly alleviated (Fig. 3). After the obturator had been polished, the finished prosthesis was placed in the patient's mouth, and was seen to provide enough retention and stability to improve her chewing capacity and comfort.

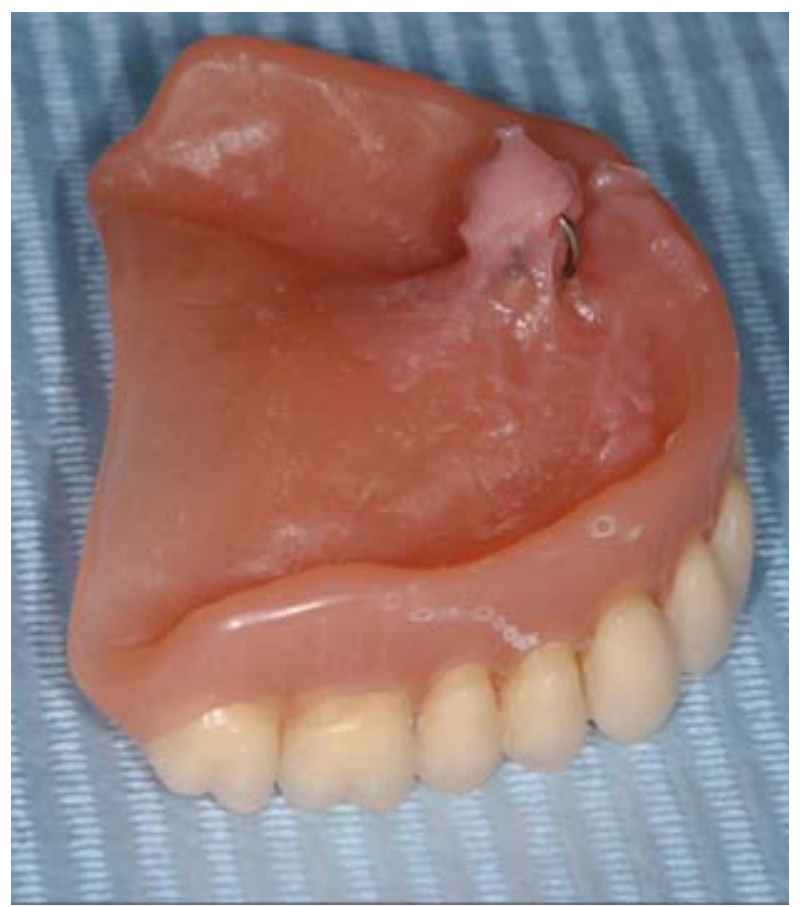

Fig. 3. A lateral view of the finished denture with the hard palatal obturator.

\section{Discussion}

The type of material to be used to create obturator prostheses depends on several factors, such as the type and extent of the cleft palate and the ability of the patient to cope with conventional removable dentures, although the aim is always to restore the anatomical loss of hard and soft tissues, providing enough retention to the denture to improve functional and psychological wellbeing. Generally, palatal obturators are used as simple solutions for the reconstruction of minor palatal defects, while larger maxillary-palatal defects represent a considerable challenge for functional and aesthetic reconstruction (6).

An obturator is indicated for cleft palate patients when there is a need to improve the retention of conventional prostheses. We believe that the use of a soft obturator is mainly indicated when the palatal defect is highly retentive, or when such a defect is very extensive or covered by an inadequately keratinized gingiva. By contrast, rigid obturators should only be used for small defects, covered by keratinized gingiva.

Prosthetic rehabilitation with a palatal obturator is mainly indicated when the closure of the fissure is not complete after corrective surgery. Proper planning, coupled with early intervention, will result in aesthetic and functional restoration for patients with a cleft palate. The use of a palatal obturator restores patient function, aesthetics and well-being. In cases for which prosthetic rehabilitation is indicated, patients can be treated by dentists trained in prosthodontics. It seems that changes related to aesthetics, function and psychological well-being has an impact on patients' personal lives and this is also a great satisfaction for care-givers (7).

In general, edentulous patients with a cleft lip and a palatal defect show a lack of retention of obturator prostheses due to their weight and the inability to achieve a proper sealing of the defect. Osseointegrated implants have been a successful alternative, allowing dentists to provide satisfactory prosthetic rehabilitation in comparison with conventional treatments (8-10). Dental implants, when properly prescribed, have been increasingly used as an alternative to conventional dentures and provide an improvement in the stability, retention, and function of the prosthesis.

Although the placement of implants in the maxilla may involve difficulties, the implants are very effective at promoting denture support, retention and stability, even in extremely atrophied edentulous maxillae, through zygomatic implants (11). Thus, the use of implants in patients with a cleft lip/palate is a valuable option because of the difficulty involved in elaborating well-fitting conventional dentures (12), since the anatomy of the residual maxillary alveolar ridge may provide little support for a conventional prosthesis. This is why an obturator appendix is used to seal the oronasal communication and increase retention.

After implant placement, rehabilitation may be performed with implant-supported fixed or removable dentures. Selection of the type of prosthesis depends on the maxillo-mandibular antero-posterior relationship, bone conditions, cost, and the individual possibility of oral hygiene... (13). However, the protocol discussed here should be enough for patients with anatomical or financial limitations to implant rehabilitation, and should be known by general practioners. Moreover, prosthetic rehabilitation with a conventional prosthesis is a cheaper and more conservative treatment than implant-supported prostheses, which are more invasive and have a higher incidence of clinical complications than conventional removable prostheses (14). Future efforts should be di- 
rected towards assessing the oral health-related quality of life of conventional and implant-retained dentures in this type of patient.

\section{Acknowledgements}

The authors are grateful to Professors José Maroto and Ramón del Castillo for the insights shared during the treatment of this patient in the recently discontinued Master's Degree in Prosthodontics of the University of Granada.

\section{References}

1. Slayton RL, Williams L, Murray J, Wheeler JJ, Lidral AC, Nishimura CJ. Genetic Association Studies of Cleft Lip and/or Palate with Hypodontia Outside the Cleft Region. Cleft Palate-Craniofac J. 2003; 40: 274-9.

2. Abadi B, Johnson JD. The prosthodontic management of cleft palate patients.J Prosthet Dent. 1982; 48: 297-302.

3. Hickey AJ, Salter M. Prosthodontic and psychological factors in treating patients with congenital and craniofacial defects. J Prosthet Dent. 2006; 95: 392-6.

4. Watanabe I, Kurtz KS, Watanabe E, Yamada M, Yoshida N, Miller AW. Multi-unit fixed partial denture for a bilateral cleft palate patient: a clinical report. J Oral Rehabil. 2005; 32: 620-2.

5 Reisberg DJ. Dental and prosthodontic care for patients with cleft or craniofacial conditions. Cleft Palate Craniofac J. 2000; 37: 534-7.

6. MacCarthy D, Murphy N. Replacement of an obturador section of an existing two-piece implant-retained edentulous obturator. J Prosthet Dent. 2000; 83: 652-5.

7. Mese A, Ozdemir E. Removable partial denture in a cleft lip and palate patient: a case report. J Korean Med Sci. 2008; 23: 924-7.

8. Harrison JW. Dental implants to rehabilitate a patient with an unrepaired complete cleft of the hard and soft palate: a clinical report. Cleft Palate Craniofac J. 1992; 29: 485-8.

9. De Rezende ML, Amado FM. Osseointegrated implants in the oral rehabilitation of a patient with cleft lip and palate and ectodermal dysplasia: a case report. Int J Oral Maxillofac Implants. 2004; 19: 896-900.

10. Abreu A, Levy D, Rodriguez E, Rivera I. Oral rehabilitation of a patient with complete unilateral cleft lip and palate using an implantretained speech-aid prosthesis: clinical report. Cleft Palate Craniofac J. 2007; 44: 673-7.

11. Pham AV, Abarca M, De Mey A, Malevez C. Rehabilitation of a patient with cleft lip and palate with an extremely edentulous atrophied posterior maxilla using zygomatic implants: case report. Cleft Palate Craniofac J. 2004; 41: 571-4.

12. Lund TW, Wade M.Use of osseointegrated implants to support a maxillary denture for a patient with repaired cleft lip and palate.Cleft Palate Craniofac J. 1993; 30: 418-20.

13. DeBoer J. Edentulous implants: overdenture versus fixed. J Prosthet Dent. 1993; 69: 386-90.

14. Fukuda M, Takahashi T, Yamaguchi T, Kochi S, Inai T, Watanabe M, Echigo S. Dental rehabilitation using endosseous implants and orthognathic surgery in patients with cleft lip and palate: report of two cases. J Oral Rehabil. 2000; 27: 546-51.

23. 